\title{
Desenvolvimento e qualidade de mudas de Parkia gigantocarpa Ducke (Fabaceae) em função de fertilizante de liberação controlada
}

Development and quality of Parkia gigantocarpa Ducke (Fabaceae) seedlings using controlledrelease fertilizer

\author{
V. P. Oliveira; R. S. Mendes; W. B. R. Martins; E. A. Santos; D. G. Araújo; M. \\ A. P. Gama*
}

Instituto de Ciências Agrárias (ICA), Universidade Federal Rural da Amazonia (UFRA), Av. Presidente Tancredo Neves, 2501, 66617-440, Belém-PA, Brasil

*email: gama_map@yahoo.com.br

(Recebido em 03 de junho de 2020; aceito em 03 de agosto de 2021)

\begin{abstract}
A Parkia gigantocarpa pertencente ao grupo das espécies pioneiras e demandantes de luz, possuindo como principal característica ecológica o crescimento rápido, sendo indicada para plantio em clareiras e recomposição de áreas em projetos de restauração florestal. O objetivo desse estudo foi avaliar os efeitos de doses crescentes do fertilizante de liberação controlada (FLC) no desenvolvimento inicial de mudas de P. gigantocarpa. O estudo foi conduzido em casa de vegetação com $50 \%$ de sombreamento, por um período de 90 dias após o transplantio das mudas. Foi utilizado o delineamento inteiramente casualizado com quatro tratamentos e dezoito repetições. Os tratamentos consistiram em quatro doses de $\operatorname{Ossmocote}^{\circledR}(0-4,1-8,2$ e 12,3 $\mathrm{g} \mathrm{dm}^{-3}$ ) na formulação NPK (15-09-12). Os resultados demostraram que a maioria dos parâmetros fitométricos e as trocas gasosas responderam de forma significativa para as doses intermediárias do FLC, podendo ser confirmados pelos aumentos em número de folhas (NF), área foliar (AF), massa seca total (MST), índice de qualidade de Dickson (IQD) e taxa fotossintética líquida $\left(P_{\mathrm{N}}\right)$. Adicionalmente, também houve reduções significativas para a concentração intercelular de $\mathrm{CO}_{2}\left(C_{\mathrm{i}}\right)$, demostrando que as mudas apresentaram maior eficiência estomática e no aparato fotossintético. Assim, o FLC contribuiu positivamente no crescimento inicial das mudas de $P$. gigantocarpa, sendo recomendado a dose de $4,1 \mathrm{~g}$ $\mathrm{dm}^{-3}$ do Osmocote ${ }^{\circledR}, 50 \%$ da dose recomenda pelo fabricante.
\end{abstract}

Palavras-chave: espécies nativas, Osmocote ${ }^{\circledR}$, trocas gasosas.

Parkia gigantocarpa belongs to the group of pioneer and light-demanding species and their main ecological characteristic is rapid growth, and this species is highly recommended for gap recovery and forest restoration projects. This study aimed to investigate the effects of increasing doses of controlled-release fertilizer (CRF) during the initial development of $P$. gigantocarpa seedlings. The study was conducted in a greenhouse under $50 \%$ shade conditions, for a period of 90 days after the seedlings were transplanted. A completely randomized design with four treatments and eighteen repetitions was used. The treatments consisted of four doses of Osmocote ${ }^{\circledR}\left(0-4.1-8.2\right.$ and $\left.12.3 \mathrm{~g} \mathrm{dm}^{-3}\right)$ in the NPK formulation (15-09-12). The results showed that most of the phytometric parameters and gas exchanges responded significantly well to intermediate doses of CRF, which could be confirmed by the increases in leaf number $(\mathrm{LN})$, leaf area $(\mathrm{LA})$, total dry mass (TDM), Dickson's quality index (DQI), and net photosynthetic rate $\left(P_{\mathrm{N}}\right)$. Furthermore, significant reductions in intercellular $\mathrm{CO}_{2}$ concentration $\left(C_{i}\right)$, were also noted showing that the seedlings displayed higher stomatal and photosynthetic apparatus efficiency. Thus, CRF contributed positively to the initial growth of $P$. gigantocarpa seedlings, and a dose of $4.1 \mathrm{~g} \mathrm{dm}^{-3}$ of Osmocote ${ }^{\circledR}$, which is $50 \%$ of the dose recommended by the manufacturer.

Keywords: native species, Osmocote ${ }^{\mathbb{R}}$, gas exchange.

\section{INTRODUÇÃO}

As florestas tropicais abrigam aproximadamente dois terços de toda a biodiversidade do planeta [1], apresentando espécies endêmicas, uma enorme diversidade de recursos genéticos e desempenham um importante papel no ciclo global do carbono [2,3]. Por outro lado, o modelo de desenvolvimento econômico para essa região apresenta inúmeros passivos ambientais, especialmente pelos impactos diretos e indiretos das atividades voltadas para a agricultura, 
pecuária, extração madeireira ilegal e mineração [4]. As alterações biofísicas, causadas por essas atividades devem ser mitigadas por meio da restauração ecológica, especialmente por meio do plantio de espécies com alto vigor fisiológico e de rápido recobrimento do solo.

Atualmente, o Brasil possui uma meta nacional de restaurar 12 milhões de hectares até o ano de 2030, estabelecida pelo Plano Nacional de Recuperação da Vegetação Nativa (PLANAVEG) $[5,6]$. Uma alternativa viável para a recuperação de áreas degradadas e para a produção de mudas é a Parkia gigantocarpa Ducke, conhecida popularmente como faveira, fava-barriguda, favaatanã ou visgueiro [7]. P. gigantocarpa é uma espécie florestal nativa pertencente à família Fabaceae, ocorrendo naturalmente em florestas de terra firme da Amazônia brasileira [8, 9], sendo classificada como pertencente ao grupo ecológico das espécies pioneiras, intolerante à sombra, com elevada taxa de sobrevivência das mudas e crescimento rápido, podendo atingir cerca de 60 metros de altura e 1,5 metros de diâmetro [10]. No entanto, o conhecimento sobre o comportamento fisiológico e morfológico da $P$. gigantocarpa e de muitas outras espécies nativas ainda é incipiente, necessitando de estudos que possam subsidiar a produção de mudas de boa qualidade física e nutricional.

Conhecer as necessidades nutricionais ideias para cada espécie nativa, bem como as melhores dosagens de fertilizantes é relevante para reduzir os custos com esses insumos, otimizar o crescimento das plantas e, ao mesmo tempo, alcançar características satisfatórias de elevada resistência aos estresses, maior robustez e conteúdo nutricional [11]. Uma alternativa viável é o uso de fertilizantes de liberação controlada (FLC), que possuem tecnologia para disponibilizar no momento adequado os nutrientes às plantas [12] ou por determinado tempo mediante condições propícias de temperatura e umidade. Esses fertilizantes, aliados a um substrato de alta qualidade, podem contribuir para reduzir o tempo de formação das mudas, as perdas dos nutrientes por lixiviação e volatização, proporcionando diversas vantagens para o ciclo produtivo em comparação com os fertilizantes comumente utilizados [13,14]. Diversos autores comprovaram os benefícios promovidos pelos FLC, principalmente nos parâmetros fisiológicos e morfológicos das plantas, como incrementos na área foliar, aumento do diâmetro do coleto, na transpiração, fotossíntese, alocação da biomassa, conteúdo nutricional e na qualidade de mudas [15-17].

Diante desse cenário, fica evidente a necessidade de estudos que determinem uma dose padrão do FLC que proporcione os melhores aspectos fisiológicos, morfológicos e nutricionais em espécies florestais nativas durante o processo de produção de mudas. Assim, o objetivo desse estudo foi avaliar o efeito de diferentes doses do FLC Osmocote ${ }^{\circledR}$ no desenvolvimento inicial de P. gigantocarpa.

\section{MATERIAL E MÉTODOS}

\section{1 Área de estudo}

O experimento foi desenvolvido na Universidade Federal Rural da Amazônia, em Belém do Pará, Brasil ( $\left(1^{\circ} 27^{\prime} 12.6^{\prime \prime S}\right.$ e 48 26'33.5"W), sendo conduzido em casa de vegetação com cobertura plástica e lateral com sombrite a 50\%, localizado no Instituto de Ciências Agrárias (ICA), por um período de 90 dias. O clima da região é do tipo Af, segundo classificação de Köppen-Geiger [18], caracterizado por apresentar temperatura média mensal superior a $18{ }^{\circ} \mathrm{C}$, com precipitação pluviométrica média de $2.537 \mathrm{~mm} \mathrm{ano}^{-1}$. A umidade relativa durante a condução do experimento variou de 60 a $80 \%$.

\subsection{Métodos de condução do experimento}

As sementes de $P$. gigantocarpa utilizadas no experimento foram coletadas em dez árvores matrizes localizadas na área urbana da cidade de Belém-PA. Após a coleta, procedeu-se com a quebra de dormência pelo método físico de escarificação da região basal e lateral da semente, com uso de um esmeril elétrico [19]. Em seguida, as sementes foram imersas em água destilada a $80{ }^{\circ} \mathrm{C}$ por um período de $24 \mathrm{~h}$ [15]. 
Após o tratamento das sementes, a semeadura foi realizada em bandejas plásticas com substrato orgânico e depois de sete dias, as plântulas foram transplantadas para tubetes de $280 \mathrm{~mL}$ preenchidos com substrato comercial Tropstrato ${ }^{\circledR}+$ vermiculita, na proporção $1: 1(\mathrm{v} / \mathrm{v})$, o qual foi misturado manualmente com o fertilizante de liberação controlada (FLC) Osmocote ${ }^{\circledR}$. Previamente a transferência para os tubetes, o substrato utilizado para germinação que estava aderido às raízes foi removido. Os tratos culturais das mudas consistiram em duas irrigações diárias e controle manual de pragas, por meio de vistorias diárias e catação manual quando necessário.

O delineamento experimental foi o inteiramente casualizado (DIC) com quatro tratamentos e dezoito repetições por tratamento, totalizando 72 unidades amostrais, sendo cada planta considerada uma unidade amostral. Os tratamentos consistiram de quatro doses do FLC Osmocote ${ }^{\circledR}$, sendo $0 \%, 50 \%, 100 \%$ e $150 \%$ da dose recomendada pelo fabricante, o que corresponde a: 0 (T1), 4,1 (T2), 8,2 (T3) e 12,3 (T4) $\mathrm{g} \mathrm{dm}^{-3}$, respectivamente. O FLC Osmocote ${ }^{\circledR}$ utilizado é constituído por NPK (15-09-12), 1,3\% de Magnésio (Mg), 5,9\% de Enxofre (S), 0,02\% de Boro (B), 0,05\% de Cobre (Cu), 0,46\% de Ferro (Fe), 0,06\% de Manganês (Mn), 0,02\% de Molibdênio (Mo) e $0,05 \%$ de Zinco (Zn), com tempo previsto de disponibilidade dos nutrientes de aproximadamente cinco meses.

\subsection{Avaliação dos parâmetros fitométricos}

Aos 90 dias após o transplantio, foram mensuradas/quantificadas as variáveis fitométricas, sendo elas: i) Altura total das mudas $(\mathrm{H})$ com auxílio de uma régua graduada em centímetros, a partir da superfície do solo até a gema apical da haste; ii) Diâmetro do Coleto (DC) com auxílio de um paquímetro digital; iii) contabilização do número de folhas (NF); posteriormente a estas medidas, todas as mudas foram seccionadas em parte aérea e raízes para medição da iv) área foliar (AF), onde uma amostra de 18 plantas por cada tratamento foi coletada para obtenção da AF, utilizando um medidor portátil (modelo LI-3100C, Area Meter, LI-COR ${ }^{\circledR}$ ) e; v) comprimento da raiz principal (CRP) mensurado com uma régua graduada em centímetros. Após a mensuração das variáveis fitométricas, a parte aérea e as raízes de cada planta foram colocadas em sacos de papel para secagem em estufa de circulação forçada com temperatura de $\pm 60^{\circ} \mathrm{C}$ até atingirem a massa constante. Assim, a massa seca da parte aérea (MSPA), massa seca da raiz (MSR) e massa seca total (MST) foram determinadas em uma balança analítica com precisão de $\pm 0,001 \mathrm{~g}$. Com isso, foi calculado o Î́ndice de Qualidade de Dickson (IQD) proposta por Dickson et al. (1960) [20] (Equação 1).

$$
\mathrm{IQD}=\frac{\mathrm{MST}}{\left(\frac{\mathrm{H}}{\mathrm{DC}}\right)+\left(\frac{\mathrm{MSPA}}{\mathrm{MSR}}\right)}
$$

Na qual:

MST: massa seca total (g planta $\left.{ }^{-1}\right)$;

$\mathrm{H}$ : altura total $(\mathrm{cm})$;

DC: diâmetro do coleto (mm);

MSPA: massa seca da parte aérea $\left(g\right.$ planta $\left.^{-1}\right)$ e;

MSR: Massa seca da raiz $\left(\mathrm{g}\right.$ planta $\left.^{-1}\right)$.

\subsection{Avaliação das trocas gasosas}

Aos 90 dias foram avaliados os parâmetros fisiológicos, utilizando o terço médio das folhas completamente expandidas. As medições foram realizadas entre o intervalo das 09:00 e 12:00 horas, em condições ambientais mais favoráveis. Utilizou-se o analisador de gás infravermelho (IRGA, modelo LI-6400XT, Versão 6.3.2, LI-COR ${ }^{\circledR}$, LincoLn, Nebraska, EUA) em níveis constantes de luz $\left(1000 \mu \mathrm{mol}\right.$ de fótons $\left.\mathrm{m}^{-2} \mathrm{~s}^{-1}\right), \mathrm{CO}_{2}(400 \mu \mathrm{mol})$ e a temperatura ambiente de 28 ${ }^{\circ} \mathrm{C}$. Para as trocas gasosas foram avaliadas a taxa fotossintética líquida $\left(P_{\mathrm{N}}\right)$, a taxa de transpiração 
$(E)$, a condutância estomática $\left(g_{s}\right)$ e a concentração intercelular de $\mathrm{CO}_{2}\left(C_{i}\right)$. A Eficiência no uso da água (WUE) foi estimada de acordo com o protocolo descrito por Ma et al. (2004) [21] que consiste na divisão da $P_{\mathrm{N}}$ por $E$. Adicionalmente, a eficiência instantânea da carboxilação $\left(P_{\mathrm{N}} / C_{\mathrm{i}}\right)$ foi calculada de acordo com metodologia descrita por Aragão et al. (2012) [22].

\subsection{Análise estatística}

Todas as variáveis foram submetidas ao teste de normalidade de Shapiro-Wilk $(p>0,05)$ e homogeneidade de variância de Levene $(p>0,05)$. Atendidos os pressupostos de normalidade e homogeneidade, procedeu-se com a análise de variância, sendo que, em caso de diferença estatística $(p<0,05)$, as médias dos tratamentos foram comparadas pelo teste Tukey $(p<0,05)$. Para as análises foi utilizado o software estatístico R, versão 3.6.3, utilizando o pacote ExpDes.pt [23].

\section{RESULTADOS}

\subsection{Crescimento e desenvolvimento inicial}

As mudas de $P$. gigantocarpa submetidas a níveis crescentes de FLC Osmocote ${ }^{\circledR}$, apresentaram aumentos em altura $(\mathrm{H})$ para os tratamentos $\mathrm{T} 2$ e T3 quando comparado ao tratamento controle (T1), no entanto, estes não diferiram significativamente entre si. Por outro lado, o T4 proporcionou redução de $11 \%$ em comparação com o T1 e diferiu $\left(\mathrm{F}_{3: 68}=4,15\right.$, $p<0,0092$ ) apenas do T3 (Tabela 1). Adicionalmente, para o DC foram detectados aumentos de $12 \%$ e $15 \%$ para T2 e T3, respectivamente, sendo observado redução significativa $\left(\mathrm{F}_{3: 68}=8,05\right.$, $p<0,0001)$ quando se utilizou o T4 comparativamente às doses de 4,1 e $8,2 \mathrm{~g} \mathrm{dm}^{-3}$.

Tabela 1: Parâmetros fitométricos para mudas de Parkia gigantocarpa aos 90 dias submetidas a doses crescentes de FLC Osmocote ${ }^{\circledR}$.

\begin{tabular}{cllllll}
\hline TRAT. & $\mathbf{H}(\mathbf{c m})$ & $\mathbf{D C}(\mathbf{m m})$ & $\mathbf{H} / \mathbf{D C}$ & $\mathbf{N F}$ & $\mathbf{A F}\left(\mathbf{c m}^{2}\right)$ & $\mathbf{C R P}(\mathbf{c m})$ \\
\hline T1 & $17,80 \pm 2,26 \mathrm{ab}$ & $5,15 \pm 0,59 \mathrm{ab}$ & $3,47 \pm 0,54 \mathrm{a}$ & $3,78 \pm 1,12 \mathrm{~b}$ & $227,09 \pm 36,42 \mathrm{~b}$ & $17,41 \pm 1,84 \mathrm{a}$ \\
T2 & $18,12 \pm 2,10 \mathrm{ab}$ & $5,79 \pm 0,87 \mathrm{a}$ & $3,19 \pm 0,55 \mathrm{a}$ & $4,94 \pm 0,62 \mathrm{a}$ & $419,12 \pm 106,09 \mathrm{a}$ & $18,09 \pm 2,57 \mathrm{a}$ \\
T3 & $18,73 \pm 2,64 \mathrm{a}$ & $5,91 \pm 1,04 \mathrm{a}$ & $3,26 \pm 0,68 \mathrm{a}$ & $4,78 \pm 0,71 \mathrm{a}$ & $419,51 \pm 113,34 \mathrm{a}$ & $16,92 \pm 5,08 \mathrm{a}$ \\
T4 & $15,89 \pm 2,40 \mathrm{~b}$ & $4,65 \pm 0,77 \mathrm{~b}$ & $3,51 \pm 0,76 \mathrm{a}$ & $5,17 \pm 0,96 \mathrm{a}$ & $197,05 \pm 101,83 \mathrm{~b}$ & $15,07 \pm 5,47 \mathrm{a}$ \\
\hline
\end{tabular}

Colunas com letras diferentes indicam diferenças significativas pelo teste de Tukey $(p<0,05)$, entre valores médios, com respectivos desvios-padrão. T1: 0 , T2: 4,1, T3: 8,2 e T4: 12,3 $\mathrm{g} \mathrm{dm}^{-3}$.

As mudas apresentaram aumentos significativos $\left(\mathrm{F}_{3: 68}=7,92, p<0,0001\right)$ para $\mathrm{NF}$ de $31 \%, 26 \%$ e 37\%, nos tratamentos T2, T3 e T4, respectivamente, em comparação com o T1 (Tabela 1). Além disso, as mudas apresentaram aumentos significativos de $84 \%$ para AF nas doses de 4,1 e 8,2 g $\mathrm{dm}^{-3}$ de FLC. Não foram observadas diferenças significativas para as variáveis H/DC e CRP.

\subsection{Influência nas trocas gasosas}

O FLC Osmocote ${ }^{\circledR}$ proporcionou resultados positivos nas trocas gasosas (Figura 1). Os valores de $P_{\mathrm{N}}, E$ e $\mathrm{g}_{\mathrm{s}}$ aumentaram significativamente $(p<0,0001)$ em mudas de $P$. gigantocarpa em função dos tratamentos T2 e T3, em comparação com o tratamento controle (Figura 1B, C, D). Por outro lado, observou-se fortes reduções de $33 \%, 23 \%$ e $52 \%$, respectivamente para o tratamento contendo a dose máxima de FLC, quando comparado ao tratamento controle (Figura 1B, C, D). Em relação a $C_{\mathrm{i}}$ foi reportado reduções significativas $\left(\mathrm{F}_{3: 68}=87,25, p<0,0001\right)$ de $27 \%$ e $26 \%$ para $\mathrm{T} 2$ e $\mathrm{T} 3$ respectivamente (Figura 1A), quando comparado ao T1. 
As mudas de $P$. gigantocarpa submetidas ao FLC não responderam de forma significativa para nenhuma das doses utilizadas quando se considerou a variável WUA (Figura 2A). Em relação a $P_{\mathrm{N}} / C_{\mathrm{i}}$, as mudas submetidas aos tratamentos $\mathrm{T} 2$ e $\mathrm{T} 3$ apresentaram aumentos significativos $\left(\mathrm{F}_{3: 68}=140,87, p<0,0001\right)$ e diferentes entre si, com acréscimos de $83 \%$ e $68 \%$, respectivamente. Adicionalmente, o T4, com a dose máxima do FLC, proporcionou comportamento similar ao tratamento controle (Figura 2B).

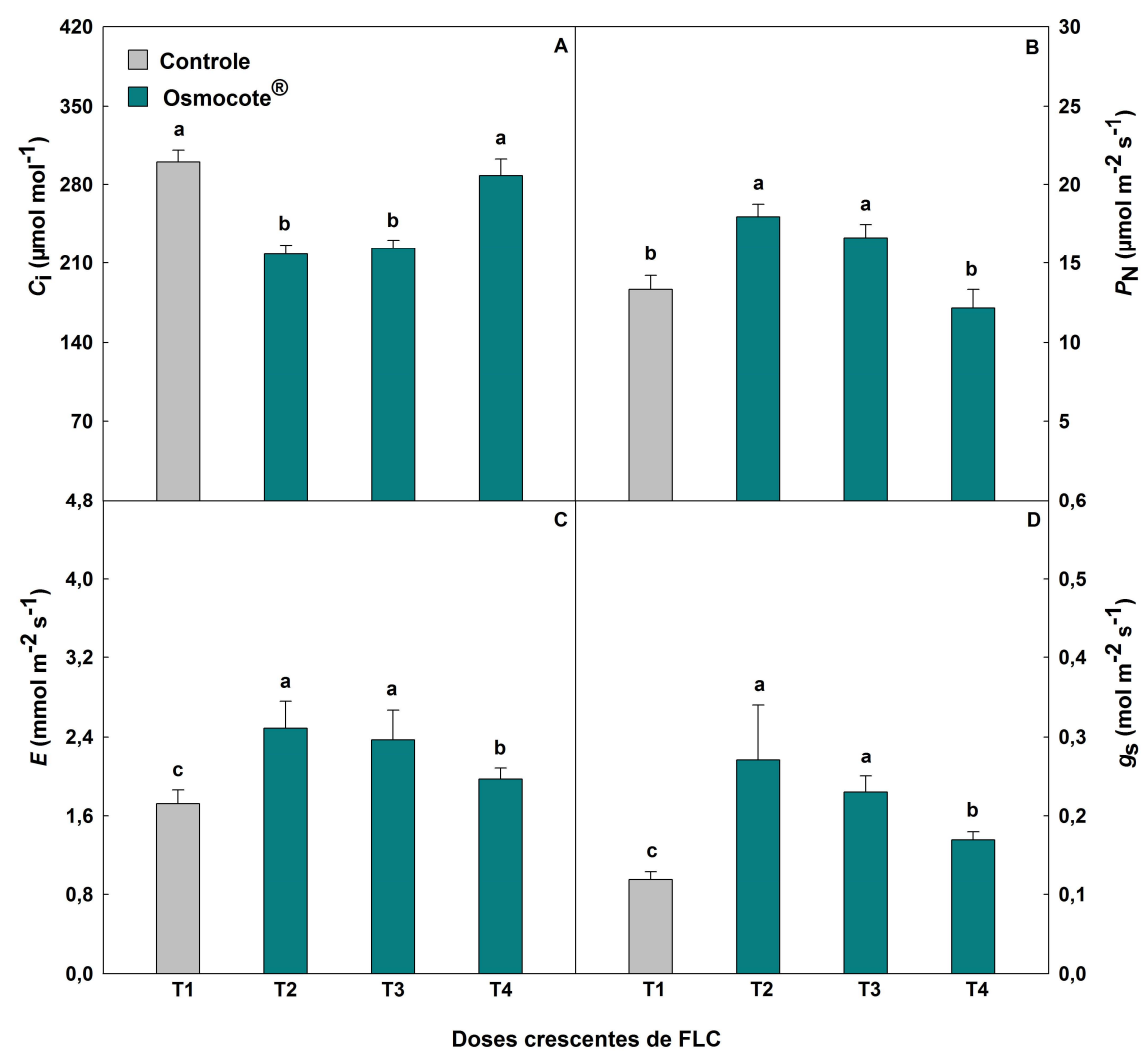

Figura 1: A: Concentração intercelular de $\mathrm{CO}_{2}\left(C_{i}\right)$; $B$ : Taxa fotossintética líquida $\left(P_{N}\right)$; $C$ : Taxa de transpiração (E); D: Condutância estomática $\left(g_{s}\right)$ em mudas de Parkia gigantocarpa aos 90 dias submetidas a doses crescentes de FLC Osmocote ${ }^{\circledR}$. Barras com letras diferentes indicam diferenças significativas pelo teste de Tukey $(p<0,05)$, entre valores médios e respectivos desvios-padrão.

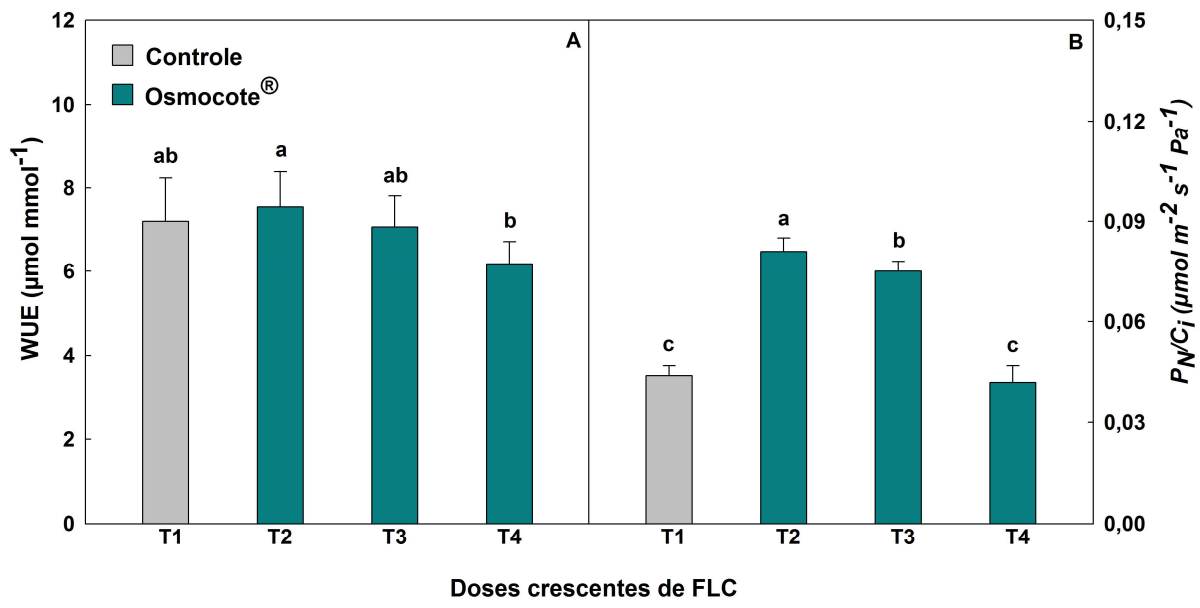

Figura 2: A: Eficiência no uso da água (WUE) e B: Eficiência instantânea da carboxilação $\left(P_{N} / C_{i}\right) \mathrm{em}$ mudas de Parkia gigantocarpa aos 90 dias, submetidas a doses crescentes de FLC Osmocote ${ }^{\circledR}$. Barras com letras diferentes indicam diferenças significativas pelo teste de Tukey $(p<0,05)$, entre valores médios e respectivos desvios-padrão. 


\subsection{Biomassa e IQD}

O FLC Osmocote ${ }^{\circledR}$ promoveu aumentos significativos $(p<0,0001)$ para a maioria dos parâmetros de biomassa, como para MSPA, MST e relação MSPA/MSR, quando utilizados os tratamentos T3 e T4 (Tabela 2). Por outro lado, a maior dose do FLC proporcionou reduções significativas $\left(\mathrm{F}_{3: 68}=21,47, p<0,0001\right)$ na MSR, quando comparada ao T1 e nos valores de MSPA e MST quando comparadas com as doses de T2 e T3. Em função disso, observou-se que a relação MSPA/MSR foi significativamente maior $\left(\mathrm{F}_{3: 68}=13,34, p<0,0001\right)$ nos tratamentos $\mathrm{T} 3$ e $\mathrm{T} 4$, quando comparados com o T1. Em relação a qualidade das mudas, avaliada pelo IQD, observouse o efeito significativo do FLC, já que foram registrados aumentos $\left(\mathrm{F}_{3: 68}=15,12, p<0,0001\right)$ nesse índice nas doses intermediárias, já o T4 apresentou redução de 65\% quando comparado ao T2.

Tabela 2: Conteúdo de massa seca e índice de qualidade de Dickson para mudas de Parkia gigantocarpa aos 90 dias após transplantio submetidas a doses crescentes de FLC Osmocote ${ }^{\mathbb{B}}$.

\begin{tabular}{clllll}
\hline TRAT. & MSR $\left(\right.$ g planta $\left.^{-1}\right)$ & MSPA $\left(\right.$ g planta $\left.^{-1}\right)$ & MSPA/MSR & MST (g planta $\left.{ }^{-1}\right)$ & IQD \\
\hline T1 & $0,84 \pm 0.19 \mathrm{a}$ & $2,55 \pm 0,42 \mathrm{~b}$ & $3,23 \pm 0,75 \mathrm{c}$ & $3,39 \pm 0,53 \mathrm{bc}$ & $0,51 \pm 0,11 \mathrm{~b}$ \\
T2 & $1,16 \pm 0,45 \mathrm{a}$ & $4,40 \pm 1,47 \mathrm{a}$ & $3,99 \pm 1,00 \mathrm{bc}$ & $5,55 \pm 1,87 \mathrm{ab}$ & $0,77 \pm 0,32 \mathrm{a}$ \\
T3 & $1,07 \pm 0.25 \mathrm{a}$ & $4,78 \pm 1,52 \mathrm{a}$ & $4,89 \pm 1,25 \mathrm{ab}$ & $5,84 \pm 1,88 \mathrm{a}$ & $0,72 \pm 0,32 \mathrm{a}$ \\
T4 & $0,39 \pm 0,17 \mathrm{~b}$ & $2,07 \pm 0,93 \mathrm{~b}$ & $5,62 \pm 1,45 \mathrm{a}$ & $2,46 \pm 1,08 \mathrm{c}$ & $0,27 \pm 0,14 \mathrm{c}$ \\
\hline
\end{tabular}

Colunas com letras diferentes indicam diferenças significativas pelo teste de Tukey $(p<0,05)$, entre valores médios e respectivos desvios-padrão. T1: $0, \mathrm{~T} 2: 4,1, \mathrm{~T} 3: 8,2 \mathrm{e} \mathrm{T} 4: 12,3 \mathrm{~g} \mathrm{dm}^{-3}$.

\section{DISCUSSÃO}

\subsection{O FLC contribuiu com o crescimento das mudas}

Os FLC são cada vez mais utilizados em viveiros florestais por reduzirem os custos com fertilizantes e permitirem um melhor aproveitamento dos nutrientes pelas plantas. Esse processo é possível devido a uma sincronia entre a liberação dos nutrientes e as necessidades nutricionais das plantas, ou seja, a taxa máxima de liberação dos nutrientes correspondem a taxa máxima de absorção pelas plantas [12]. Esses fertilizantes possuem alta tecnologia, principalmente, pelo fato de que os nutrientes são encapsulados e revestidos por polímeros, o que permite a liberação gradual dos nutrientes [24, 25]. Ressalta-se ainda que esses fertilizantes diminuem os passivos ambientais, principalmente por reduzirem as emissões dos gases do efeito estufa e diminuírem a eutrofização dos cursos d'água [26], já que essa liberação gradual de nutrientes ocorre, à princípio, de acordo com o desenvolvimento e necessidade da planta, não ocorrendo lixiviação e volatização dos nutrientes.

As doses intermediárias de Osmocote $^{\circledR}$ influenciaram positivamente nos parâmetros fitométricos das mudas de $P$. gigantocarpa aos 90 dias de experimento. Os resultados desse estudo, demostram que as doses 4,1 e $8,2 \mathrm{~g} \mathrm{dm}^{-3}$ promoveram suaves ganhos para $\mathrm{H}$ e DC, além de incrementos significativos para NF e AF, sendo esses quatros parâmetros importantes na qualidade e na redução do tempo das mudas em viveiro. H e o DC são variáveis fitométricas potencialmente importantes, por indicarem a capacidade de maior sobrevivência das mudas no sítio, bem como conferirem rusticidade às espécies [27, 28]. Nesse estudo, o uso de FLC proporcionou maior AF para as mudas, sendo uma característica provavelmente relacionada à uma maior capacidade do aparato fotossintético, principalmente por aumentar a produção de fotoassimilados pela espécie [29].

Embora não tenha sido observada diferenças significativas para CRP (Tabela 1), essa é uma variável que está relacionada à qualidade do substrato utilizado, de modo que a presença do FLC provavelmente não alterou as características físicas, como aeração, estrutura e retenção de água pelo substrato, permitindo desenvolvimento equilibrado do sistema radicular das mudas. Silva et 
al. (2019) [30] avaliando o efeito de doses crescentes do Osmocote Plus ${ }^{\circledR}$ em mudas de Acacia mangium destinadas a biomassa, reportaram incrementos em $\mathrm{DC}$ e $\mathrm{AF}$, quando comparadas ao tratamento sem a aplicação do FLC. Assim como Dutra et al. (2016) [31], que apontaram melhores resultados para o crescimento e padrão de qualidade das mudas Peltophorum dubium quando submetidas à doses entre $5,4 \mathrm{a} 8,2 \mathrm{~g} \mathrm{dm}^{-3}$ de FLC Osmocote ${ }^{\mathbb{R}}$ em relação ao tratamento controle.

\subsection{Repercussão do FLC nas trocas gasosas}

Mudas de $P$. gicantocarpa submetidas ao FLC Osmocote ${ }^{\circledR}$ apresentaram aumentos em $P_{\mathrm{N}}, E$, $g_{\mathrm{s}}$, WUE e $P_{\mathrm{N}} / C_{\mathrm{i}}$. Esses efeitos positivos indicam uma melhoria substancial no desempenho estomático, como pode ser confirmado pelos aumentos reportados em $g_{\mathrm{s}}$ nos tratamentos com 4,1, 8,2 e $12,3 \mathrm{~g} \mathrm{dm}^{-3}$ (Figuras 1 e 2). Os incrementos promovidos para a $P_{\mathrm{N}}$ podem ser corroborados principalmente pelo fato de que as mudas detiveram um maior desenvolvimento em $\mathrm{AF}$, indicando melhorias e eficiência no processo fotossintético. Em nosso estudo, embora tenha ocorrido toxidade pelo uso da dose máxima do FLC no T4, ainda reportamos melhores resultados em $g_{\mathrm{s}}$ e $E$ quando comparado ao T1. Esses resultados podem ser explicado pelos ajustes fisiológicos das mudas em um primeiro momento, repercutindo positivamente nessas variáveis quando comparado as mudas do T1 que não foram submetidas ao FLC. Adicionalmente, não encontramos diferenças significativas para os valores de WUE em mudas com FLC Osmocote ${ }^{\circledR}$, demostrando que independente da dose utilizada, o substrato promoveu maior absorção de água pelas raízes das plantas [32].

Os aumentos encontrados em $P_{\mathrm{N}} / C_{\mathrm{i}}$ e a redução simultânea para $C_{\mathrm{i}}$ podem ser explicados pela maior atividade da enzima $\mathrm{RuBisCO}$, pois o processo de fotossíntese é dependente da assimilação de $\mathrm{CO}_{2}$ atmosférico, no qual, em condições ambientais favoráveis, reduz $C_{\mathrm{i}}[33,34]$. Tian et al. (2017) [35] estudando o efeito do FLC à base de potássio em mudas de Gossypium hirsutum, reportaram incrementos no desempenho fotossintético quando comparado ao uso de fertilizante convencional. Dong et al. (2016) [36] utilizando um FLC, com formulação NPK (15-6-12) em plantas de Zea mays por 90 dias, verificaram aumentos significativos para o conteúdo de clorofila, $P_{\mathrm{N}}, E$ e para os parâmetros de fluorescência das clorofilas quando comparadas ao tratamento controle. Smiderle et al. (2020) [37] testando cinco doses de FLC em mudas de Annona cacans por um período de 180 dias encontraram aumentos nos pigmentos fotossintéticos, demostrando influência direta do fertilizante para esse parâmetro.

\subsection{Benefícios do FLC na biomassa e IQD}

O FLC Osmocote ${ }^{\circledR}$ impactou positivamente no conteúdo de biomassa das mudas de $P$. gicantocarpa, induzindo incrementos significativos em MSR, MSPA e MST. Esses aumentos estão intimamente ligados aos benefícios observados nas trocas gasosas e na maior capacidade fotossintética das folhas devido os incrementos em AF. Os tratamentos T2 e T3 proporcionaram incrementos significativos para o IQD, quando comparado ao tratamento controle. Além disso, a dose máxima utilizada do FLC inibiu fortemente os parâmetros de biomassa no T4, demostrando certa toxidez para a espécie. O IQD é um índice bastante utilizado para identificar e selecionar mudas de qualidade e adequadas para o plantio em sítio, especialmente por incluir parâmetros como o cálculo de robustez e balanço de distribuição da biomassa aérea e das raízes [38]. No entanto, ressaltamos que esse índice não pode ser analisado isoladamente pois apresenta determinada fragilidade, pois não engloba todos os requisitos necessários para se atestar uma boa produção, como por exemplo a qualidade de germinação das sementes, infestação por patógenos e doenças [39]. Da Silva et al. (2019) [40] investigando o efeito do FLC no crescimento e qualidade de mudas de Inga heterophylla verificaram diferenças significativas para o IDQ, sendo recomendada uma dose de $6,2 \mathrm{~g} \mathrm{dm}^{-3}$ para essa espécie. Adicionalmente, Souza et al. (2020) [41] comparando diferentes substratos com ausência e presença do FLC para a produção de mudas de Agonandra brasiliensis, reportaram aumentos para MSR e MSPA em todos os tratamentos com FLC, em especial a combinação do substrato areia + Forth Cote $^{\circledR}$. 


\section{CONCLUSÃO}

De acordo com as condições experimentais avaliadas nesse estudo, o crescimento das mudas de $P$. gigantocarpa é influenciado pela liberação controlada do fertilizante. Recomendamos para a produção de mudas desta espécie a dose de $4,1 \mathrm{~g} \mathrm{dm}^{-3}$ do FLC Osmocote ${ }^{\circledR}$, visto que os efeitos benéficos são similares a dose de $8,2 \mathrm{~g} \mathrm{dm}^{-3}$. Além disso, haverá uma redução considerável dos custos no processo produtivo. Ademais, destacamos o potencial ecológico da espécie para produção em larga escala em viveiros florestais visando a restauração florestal de ecossistemas antropizados.

\section{AGRADECIMENTOS}

A Hydro pelo apoio financeiro ao Consórcio de Pesquisa em Biodiversidade Brasil-Noruega (BRC). Ao Conselho Nacional de Desenvolvimento Científico e Tecnológico (CNPq), pela concessão de bolsa de pós-graduação na modalidade mestrado acadêmico ao primeiro autor (134262/2019-0). E aos revisores anônimos pelas substanciais contribuições ao longo desse artigo.

\section{REFERÊNCIAS BIBLIOGRÁFICAS}

1. Aerts R, Honnay O. Forest restoration, biodiversity and ecosystem functioning. BMC Ecol. 2011 Nov;11(1):11-29. doi: 10.1186/1472-6785-11-29

2. Gardner TA, Barlow J, Chazdon R, Ewers RM, Harvey CA, Peres CA, et al. Prospects for tropical forest biodiversity in a human-modified world. Ecol Lett. 2009 Jun;12(6):561-82. doi: 10.1111/j.14610248.2009.01294.x

3. Houghton RA, Byers B, Nassikas AA. A role for tropical forests in stabilizing atmospheric $\mathrm{CO}_{2}$. Nat Clim Chang. 2015 Nov;5(12):1022-3. doi: 10.1038/nclimate2869

4. Aide TM, Clark ML, Grau HR, López-Carr D, Levy MA, Redo D, et al. Deforestation and reforestation of Latin America and the Caribbean (2001-2010). Biotropica. 2013 Aug;45(2):262-71. doi: 10.1111/j.1744-7429.2012.00908.x

5. Ministério do Meio Ambiente. Potencial de regeneração natural da vegetação no Brasil [Internet]. Brasília (DF): MMA; 2017 [acesso em 12 fev 2020]. Disponível em: http://coral.ufsm.br/ccr/images/Potencial_de_regeneracao_natural_brasil.compressed.pdf

6. Brasil. Ministério do Meio Ambente. Planaveg: Plano nacional de recuperação da vegetação nativa [Internet]. Brasília (DF): MMA; 2017. [acesso em 12 fev 2020]. Disponível em: https://snif.florestal.gov.br/images/pdf/publicacoes/planaveg_publicacao.pdf

7. Gomes JM, de Carvalho JOP, da Silva MG, Nobre DNV, Taffarel M, Ferreira JER, et al. Sobrevivência de espécies arbóreas plantadas em clareiras causadas pela colheita de madeira em uma floresta de terra firme no município de Paragominas na Amazônia brasileira. Acta Amaz. 2010 Mar;40(1):171-8.

8. Embrapa Amazônia Oriental. Espécies arbóreas da Amazônia No 11: Fava-atanã (Parkia gigantocarpa). Belém (PA): Embrapa; 2004.

9. REFLORA. Flora do Brasil 2020 - Algas, fungos e plantas [Internet]. Rio de Janeiro: Instituto de Pesquisas Jardim Botânico do Rio de Janeiro; 2020 [acesso em 23 fev 2020]. Disponível em: http://floradobrasil.jbrj.gov.br/reflora/listaBrasil selecionando Angiospermas e Parkia gigantocarpa Ducke no menu.

10. Carvalho PER. Espécies arbóreas brasileiras. Brasília (DF): Empraba Informação Tecnológica; 2010. $644 \mathrm{p}$.

11. Caldeira MVW, Delarmelina WM, Lübe SG, Gomes DR, de Oliveira Gonçalves E, Alves AF. Biossólido na composição de substrato para a produção de mudas de Tectona grandis. Floresta. 2012 Mar;42(1):77-84.

12. Irfan SA, Razali R, KuShaari K, Mansor N, Azeem B, Ford Versypt AN. A review of mathematical modeling and simulation of controlled-release fertilizers. J Control Release. 2018 Feb;271:45-54. doi: 10.1016/j.jconrel.2017.12.017

13. Stüpp ÂM, Navroski MC, Felippe D, Kniess DDC, Amancio JC, Silva MA, et al. Crescimento de mudas de Mimosa scabrella Benth em função de diferentes tamanhos de recipientes e doses de fertilizante. Rev Ecol e Nutr Florest. 2015 Mai;3(2):40-7. 
14. Menegatti RD, Guollo K, Navroski MC, Vargas OFV. Fertilizante de liberação lenta no desenvolvimento inicial de Aspidosperma parvifolium A. DC. Sci Agrar Parana. 2017 Abr;16(1):45-9. doi: 10.18188/1983-1471/sap.v16n1p45-49

15. Rossa UB, Angelo AC, Nogueira AC, Bognola IA, Pomianoski DJW, Soares PRC, et al. Fertilização de liberação lenta no crescimento de mudas de Paricá em viveiro. Pesqui Florest Bras. 2013 Sep;33(75):227-34.

16. de Andrade Júnior WV, dos Santos Filho BG, de Oliveira Neto CF, Pereira ACC, da Silva RTL, Viégas IJM, et al. Ecophysiological and biochemical behavior in young plants of Parkia gigantocarpa Ducke subjected to waterlogging conditions. African J Agric Res. 2016 Jan;11(4):284-97. doi: 10.5897/AJAR2015.10459

17. Tian C, Zhou X, Liu Q, Peng J, Wang W, Zhang Z, et al. Effects of a controlled-release fertilizer on yield, nutrient uptake, and fertilizer usage efficiency in early ripening rapeseed (Brassica napus L.). J Zhejiang Univ B. 2016 Oct;17(10):775-86. doi: 10.1631/jzus.B1500216

18. Alvares CA, Stape JL, Sentelhas PC, de Moraes Gonçalves JL, Sparovek G. Köppen's climate classification map for Brazil. Meteorol Zeitschrift. 2013 Dec;22(6):711-28. doi: 10.1127/09412948/2013/0507

19. de Melo MGG, de Mendonça MS, Nazário P, Mendes AMS. Superação de dormência em sementes de três espécies de Parkia spp. Rev Bras Sementes. 2011 Jan;33(3):533-42.

20. Dickson A, Leaf AL, Hosner JF. Quality appraisal of white spruce and white pine seedling stock in nurseries. For Chron. 1960 Mar;36(1):10-3. doi: 10.5558/tfc36010-1

21. Ma JF, Mitani N, Nagao S, Konishi S, Tamai K, Iwashita T, et al. Characterization of the silicon uptake system and molecular mapping of the silicon transporter gene in rice. Plant Physiol. 2004 Oct;136(2):3284-9. doi: 10.1104/pp.104.047365

22. Aragão RM, Silva EN, Vieira CF, Silveira JAG. High supply of $\mathrm{NO}_{3}$ - mitigates salinity effects through an enhancement in the efficiency of photosystem II and $\mathrm{CO}_{2}$ assimilation in Jatropha curcas plants. Acta Physiol Plant. 2012 Jun;34(6):2135-43. doi: 10.1007/s11738-012-1014-y

23. R Development Core Team. R: A language and environment for statistical computing. Viena (AT): R Foundation for Statistical Computing; 2017. Disponível em: http://www.r-project.org/

24. Wei Y, Li J, Li Y, Zhao B, Zhang L, Yang X, et al. Research on permeability coefficient of a polyethylene controlled-release film coating for urea and relevant nutrient release pathways. Polym Test. 2017 May;59:90-8. doi: 1016/j.polymertesting.2017.01.019

25. Gibson EL, Gonçalves EO, dos Santos AR, Araújo EF, Caldeira MVW. Controlled-Release fertilizer on growth of Melanoxylon brauna Schott seedlings. Floresta e Ambient. 2019 Sep;26:1-7. doi: 10.1590/2179-8087.041818

26. Azeem B, KuShaari K, Man ZB, Basit A, Thanh TH. Review on materials \& methods to produce controlled release coated urea fertilizer. J Control Release. 2014 May;181(1):11-21. doi:10.1016/j.jconrel.2014.02.020

27. Freitas SJ, de Carvalho AJC, Berilli SS, dos Santos PC, Marinho CS. Substratos e Osmocote ${ }^{\circledR}$ na nutrição e desenvolvimento de mudas micropropagadas de abacaxizeiro cv. Vitória. Rev Bras Frutic. 2011 Out;33(spe1):672-9. doi: 10.1590/S0100-29452011000500094

28. Mota LHS, Scalon SPQ, Heinz R. Shading in emergency of seedlings and on the initial growth of Dipteryx alata Vog. Cienc Florest. 2012 Jul;22(3):423-31.

29. Santos CC, Motta IS, Carneiro LF, Santos MCS, Padovan MP, Mariani A. Produção agroecológica de mudas de maracujá em substratos a base de húmus de minhoca e casca de arroz carbonizada. Cad Agroecol. 2014 Feb;9(4):1-10.

30. Silva LDD, Lima APL, Lima SF, Silva RC, Paniago GF. Controlled-release fertilizer in the production and quality of Acacia mangium seedlings. Floresta e Ambient. 2019 May;26(2):1-7. doi: 10.1590/21798087.020917

31. Dutra TR, Massad MD, Sarmento MFQ. Fertilizante de liberação lenta no crescimento e qualidade de mudas de canafístula (Peltophorum dubium (Srenpg.) Taub.). Floresta. 2016 Out;46(4):491-8. doi: 10.5380/rf.v46i4.44570

32. Bhalerao SA, Sharma AS, Poojari AC. Toxicity of nickel in plants. Int J Pure Appl Biosci. 2015 Apr;3(2):345-55.

33. Yu JQ, Huang LF, Hu WH, Zhou YH, Mao WH, Ye SF, et al. A role for brassinosteroids in the regulation of photosynthesis in Cucumis sativus. J Exp Bot. 2004 May;55(399):1135-43. doi: 10.1093/jxb/erh123

34. Parry MAJ, Andralojc PJ, Scales JC, Salvucci ME, Carmo-Silva AE, Alonso H, et al. Rubisco activity and regulation as targets for crop improvement. J Exp Bot. 2013 Jan;64(3):717-30. doi: $10.1093 / \mathrm{jxb} / \mathrm{err} 313$ 
35. Tian X-F, Li C-L, Zhang M, Lu Y-Y, Guo Y-L, Liu L-F. Effects of controlled-release potassium fertilizer on available potassium, photosynthetic performance, and yield of cotton. J Plant Nutr Soil Sci. 2017 Jul;180(5):505-15. doi: 10.1002/jpln.201700005

36. Dong YJ, He MR, Wang ZL, Chen WF, Hou J, Qiu XK, et al. Effects of new coated release fertilizer on the growth of maize. J Soil Sci Plant Nutr. 2016 Set;16(3):637-49. doi: S0718-95162016005000046

37. Smiderle OJ, Souza AG, Menegatti RD. Controlled-release fertilizer in the production of seedlings of Anonna cacans Warm. J Agric Stud. 2020 Feb;8(3):125. doi: 10.5296/jas.v8i3.16163

38. Bonamigo T, Scalon SPQ, Pereira ZV. Substratos e níveis de luminosidade no crescimento inicial de mudas de Tocoyena formosa (Cham. \& Schltdl.) K. Schum. (Rubiaceae). Ciência Florest. 2016 Apr;26(2):501-11. doi: 10.5902/1980509822750.

39. Barbosa TP, Chagas JRM, Silva BO, da Silva EG, Lima TTS. Crescimento e qualidade de mudas de Schizolobium parahyba var. amazonicum em diferentes substratos. Rev Cienc Agrar. 2019 Oct;62:1-7. doi: 10.22491/rca.2019.3052.

40. da Silva EJS, Senado JAV, da Silva ÁE, Gama MAP, Ohashi ST, de Souza GMP, et al. Growth and quality of Inga heterophylla willd seedlings according to the slow release fertilizer. J Agric Sci. 2019 Apr;11(5):479-84. doi: 10.5539/jas.v11n5p479.

41. Souza AG, Smiderle OJ, Montenegro RA, Moriyama TK, Dias TJ. Controlled-Release fertiliser and substrates on seedling growth and quality in Agonandra brasiliensis in Roraima. J Agric Stud. 2020 Jan;8(3):70-80. doi: 10.5296/jas.v8i3.16088. 